\title{
Evolution and precession of accretion disk in tidal disruption events
}

\author{
R.-F. Shen ${ }^{\mathrm{a}}$ and C.D. Matzner \\ Department of Astronomy \& Astrophysics, University of Toronto, Canada
}

\begin{abstract}
In a supermassive black hole (BH) tidal disruption event (TDE), the tidally disrupted star feeds the $\mathrm{BH}$ via an accretion disk. Most often it is assumed that the accretion rate history, hence the emission light curve, tracks the rate at which new debris mass falls back onto the disk, notably the $t^{-5 / 3}$ power law. But this is not the case when the disk evolution due to viscous spreading - the driving force for accretion - is carefully considered. We construct a simple analytical model that comprehensively describes the accretion rate history across 4 different phases of the disk evolution, in the presence of mass fallback and disk wind loss. Accretion rate evolves differently in those phases which are governed by how the disk heat energy is carried away, early on by advection and later by radiation. The accretion rate can decline as steeply as $t^{-5 / 3}$ only if copious disk wind loss is present during the early advection-cooled phase. Later, the accretion rate history is $t^{-8 / 7}$ or shallower. These have great implications on the TDE flare light curve. A TDE accretion disk is most likely misaligned with the equatorial plane of the spinning BH. Moreover, in the TDE the accretion rate is super- or near-Eddington thus the disk is geometrically thick, for which case the BH's frame dragging effect may cause the disk precess as a solid body, which may manifest itself as quasi-periodic signal in the TDE light curve. Our disk evolution model predicts the disk precession period increases with time, typically as $\propto t$. The results are applied to the recently jetted TDE flare Swift transient J1644 + 57 which shows numerous, quasi-periodic dips in its long-term X-ray light curve. As the current TDE sample increases, the identification of the disk precession signature provides a unique way of measuring $\mathrm{BH}$ spin and studying $\mathrm{BH}$ accretion physics.
\end{abstract}

\section{INTRODUCTION}

Past modeling of supermassive black hole $(\mathrm{BH})$ tidal disrution events (TDEs) has often assumed the accretion rate onto the $\mathrm{BH}$ and its history are identical to those of the bound debris returns to the periastron and circularizes $[1,2]$. However, when the disk evolution due to viscosity the driving force for angular momentum transport, hence for accretion - is carefully considered, as will be shown in this contribution, that is usually not the case. More details of the work presented here are contained in a upcoming paper (Shen \& Matzner 2012, in prep.).

Previous works studying the viscous evolution of the TDE disk considered either the very late stage of the evolution, 10-100 years after the disruption [3], or only the earliest stage (for $t \sim$ the viscous time of the initial disk) of the disk evolution while artificially truncating the disk at the radius where it was initially formed [4].

\section{TDE FALLBACK}

Let us start with reviewing some characteristics of a TDE. The tidal disruption radius is defined as

$$
R_{t}=R_{*}\left(M / M_{*}\right)^{1 / 3}=23 M_{6}^{-2 / 3} m_{*}^{-1 / 3} r_{*} R_{\mathrm{Sch}},
$$

where $M=10^{6} M_{6} M_{\odot}$ is the BH mass, $M_{*}=m_{*} M_{\odot}$ and $R_{*}=r_{*} R_{\odot}$ are mass and radius of the star, respectively, and $R_{\mathrm{Sch}}=2 G M / c^{2}$ is the BH Schwarzschild radius. For a stellar orbit with the pericenter radius $R_{p}$, the "penetration" parameter is defined as $\beta=R_{t} / R_{p}$. After the disruption, about one half of the disrupted star becomes bound to the $\mathrm{BH}$ and falls successively back along eccentric orbits. The returning time for the most bound material is $[5,6]$

$$
t_{\mathrm{f}}=3.3 \times 10^{6} \beta^{-3} M_{6}^{1 / 2} r_{*}^{3 / 2} m_{*}^{-1} \mathrm{~s} .
$$

a e-mail: gf25@nyu.edu
After this time, the returning bound debris material collides with other debris material, circularizes at $R_{f}=$ $2 R_{p}$ and then accretes onto the hole.

The first-order, standard model predicts that the rate at which the mass falls back and feed the accretion disk declines as $t^{-5 / 3}$. Considering the internal structure of the star, later work show that the fallback rate history is stable up to $t_{f}$ before it declines $[1,7]$. So we approximate the overall mass fallback rate history as

$$
\dot{M}_{\mathrm{fb}}(t) \simeq \frac{M_{*}}{5 t_{\mathrm{f}}} \times \begin{cases}1, & \text { for } t \lesssim t_{\mathrm{f}}, \\ \left(\frac{t}{t_{\mathrm{f}}}\right)^{-5 / 3}, & \text { for } t>t_{\mathrm{f}} .\end{cases}
$$

The prefactor of 5 in the denominator is set such that the time-integrated fallback mass is $M_{*} / 2$. Normalized by the Eddington accretion rate $\dot{M}_{\text {Edd }}=L_{\text {Edd }} /\left(0.1 c^{2}\right)$, the peak fallback rate is $\dot{m}_{\mathrm{fb}}\left(t_{f}\right)=\dot{M}_{\mathrm{fb}}\left(t_{f}\right) / \dot{M}_{\mathrm{Edd}}=$ $87 \beta^{3} M_{6}^{-3 / 2} r_{*}^{-3 / 2} m_{*}^{2}$, which is highly super-Eddington.

\section{VISCOUS EVOLUTION OF DISK}

The surface density evolution $\Sigma(R, t)$ of a Keplerian is governed by the following viscous diffusion equation $[8,9]$

$$
\frac{\partial \Sigma}{\partial t}=\frac{3}{R} \frac{\partial}{\partial R}\left[R^{1 / 2} \frac{\partial}{\partial R}\left(R^{1 / 2} v \Sigma\right)\right]+S(R, t),
$$

where $S(R, t)$ is a source/sink term representing mass infall onto and/or mass loss from the disk. The kinetic viscosity coefficient is $v=2 \alpha P /\left(3 \Omega_{K} \rho\right)$, where $\alpha$ is the standard Shakura \& Sunyaev viscosity parameter. For $v$ depends only on radius in a power law as $v \propto R^{n}$ and for the case of the mass fall back is unimportant, i.e., $S(R, t)=0$, Eq. (4) is linear and straightforward to solve analytically. Consequently, we have the following scaling laws [10,11]

$$
\Sigma \propto R^{-n}, R_{O} \propto t^{\frac{1}{2-n}}, \text { and } \dot{M}_{\mathrm{acc}} \propto t^{-\frac{5-2 n}{4-2 n}},
$$


where $R_{o}$ is the disk outer radius. Eq. (5) suggests that $\dot{M}_{\text {acc }}$ for an initial ring decays shallower than $\dot{M}_{\mathrm{fb}}$ for $n<5 / 4$. In that case the disk accretion rate will not follow $\dot{M}_{\mathrm{fb}}(t)$.

As accretion rate drops, the change of accretion regimes changes $n$. A comprehensive understanding of $\dot{M}_{\text {acc }}$ evolution needs to consider the change of $n$, and to include the impacts of fallback and wind loss. In order to know $n$ and its evolution, we need to determine the disk structure. Following $[12,13]$, we consider both the advective and radiative cooling adopting the slim disk model [14]. The local energy equation in the disk is

$$
Q^{+}=Q_{\mathrm{adv}}^{-}+Q_{\mathrm{rad}}^{-},
$$

which states the balance between the viscous heating rate and two cooling rates: the advective cooling and the radiative cooling. If the advective cooling dominates, i.e., $Q_{\text {adv }}^{-} \gg Q_{\text {rad }}^{-}$, which is appropriate for the early stages of TDE disk evolution, it can be shown from Eq. (6) that $v \propto$ $R^{1 / 2}$, hence $n=1 / 2$. The pressure is the sum of radiation pressure and gas pressure: $P=P_{\text {rad }}+P_{\text {gas }}$. If the disk, however, is radiative-cooling dominated, i.e., $Q_{\mathrm{adv}}^{-} \ll Q_{\mathrm{rad}}^{-}$, it can be shown that $v \propto R^{-3 / 2}$ for $P \simeq P_{\text {rad }} \gg P_{\text {gas }}$, hence $n=-3 / 2$, wheas $v \propto R^{3 / 5}$ for $P \simeq P_{\text {gas }} \gg P_{\text {rad }}$, hence $n=3 / 5$. In the following, we will assume $P_{\text {rad }} \gg P_{\text {gas }}$, and also check when the gas pressure starts to dominate.

For convenience, we normalize the accretion rate $\dot{M}_{\text {acc }}$ and sizes $(H, R)$ by $\dot{M}_{\text {Edd }}$ and $R_{\text {Sch }}$, respectively, and denote normalized ones with $\dot{m}$ and $(h, r)$, respectively. Eq. (6) then can be solved for the rtio $h / r$ whose solution is $h / r \approx$ $\min (1, \dot{m} / r)$.

It follows from Eq. (6) that the ratio of two cooling terms is $Q_{\text {adv }}^{-} / Q_{\text {rad }}^{-}=(\dot{m} / r)(h / r)$. Therefore, the regime of advection-dominated accretion flow (ADAF) corresponds to high $\dot{m} / r$. Then for a given $\dot{m} \gtrsim 1$, there exists a transition radius $r_{\text {tra }}$ at which $Q_{\text {adv }}^{-} \simeq Q_{\text {rad }}^{-}$. It separates an inner ADAF region and an outer radiative cooled region. One finds $r_{\text {tra }} \simeq \dot{m}$.

The gas pressure is important only for low accretion rate and/or larger radius. Setting $P_{\text {rad }}=P_{\text {gas }}$ in Eq. (6) gives a critical radius

$$
r_{\text {gas }}=787 \dot{m}^{16 / 21}\left(\alpha_{-1} M_{6}\right)^{2 / 21},
$$

outside which $P_{\text {gas }}$ dominates over $P_{\text {rad }}$.

As the fallback rate drops with time from superEddington to sub-Eddington, the disk regime may transfer from ADAF to radiative. We thus divide the long-term disk evolution into four phases. Phase 0 is the earliest, before $t_{f}$ when $\dot{m}_{\mathrm{fb}}(t)$ starts to drop, and is in ADAF. Phase 1 is still in ADAF, but it ends at $t_{\text {tra }}$ when the outer disk edge starts to be radiative. In Phase 2, an inner ADAF region and an outer radiative region co-exist, and the separation radius $r_{\text {tra }}$ advances inward with time. Lastly, Phase 3 is when the entire disk is radiative.

An ADAF disk tends to launch powerful outflow (wind). We use a simple prescription for the disk wind loss by assuming the local accretion rate is a power law function of radius: $\dot{m}(r) \propto r^{s}$ where $0<s<1[15,16]$. We keep $s$ as time independent. One then finds $\Sigma \propto r^{-n+s}$. We assume that wind loss occurs only to the ADAF region of the disk. Moreover, the evolution of the disk outer radius $r_{o}(t)$ is essentially unaffected by accretion-powered winds.
Now we can work out the analytical scalings of the disk structure evolution, specifically the surface density profile, the local accretion rate and the disk outer radius, for the four phases.

Phase 0 is the disk "built-up" phase and the disk structure is severely shaped by the fallback. Since Phase 0 is in ADAF regime, the wind loss needs to be considered. Then we have

$$
\begin{gathered}
\Sigma(r) \propto \begin{cases}r^{-1 / 2+s}, & \text { for } r<r_{f}, \\
r^{-1}, & \text { for } r>r_{f} ;\end{cases} \\
\dot{m}(r) \simeq \begin{cases}\dot{m}_{\mathrm{fb}}(t)\left(r / r_{f}\right)^{s}, & \text { for } r<r_{f}, \\
0, & \text { for } r>r_{f} .\end{cases}
\end{gathered}
$$

The disk outer radius evolution is still given by the viscous spreading solution $r_{o} \propto t^{2 / 3}$.

In Phase 1 , the fallback rate starts to drop as $t^{-5 / 3}$. The fallback is important only when the accretion rate due to viscous spreading - after considering wind loss - drops faster than $\dot{m}_{\mathrm{fb}}$, which is possible for strong wind loss, i.e., large $s$. For weaker wind, i.e., $s<1 / 3$, the disk is described by the viscous spreading solution plus wind loss:

$$
\Sigma \propto r^{-1 / 2+s}, \quad r_{o} \propto t^{2 / 3}, \text { and } \dot{m} \propto t^{-4 / 3-s} .
$$

For stronger wind, i.e., $s>1 / 3$, the fallback becomes important, then the surface density and the accretion rate are given by Eqs. (8-9) again. The evolution of $r_{o}$ doesn't depend on $s$.

In Phase 2, the outer disk region $\left(r>r_{\text {tra }}\right)$ transitions to radiatively efficient, i.e., $n=-3 / 2$, and the inner disk region remains advective $(n=1 / 2)$. So the surface density is

$$
\Sigma \propto \begin{cases}r^{-1 / 2+s}, & r<r_{\text {tra }} \\ r^{3 / 2}, & r>r_{\text {tra }} .\end{cases}
$$

The outer disk radius is now $r_{o} \propto t^{2 / 7}$. The local accretion rate is

$$
\dot{m} \propto \begin{cases}t^{-8(1-s) / 7}, & r<r_{\text {tra }} \\ t^{-8 / 7}, & r>r_{\text {tra }} .\end{cases}
$$

Since $\dot{m}$ drops shallowly, the fallback is unimportant.

In phase 3 , the disk is completely radiative and has no wind. Thus,

$$
\Sigma \propto r^{3 / 2}, \quad r_{o} \propto t^{2 / 7}, \text { and } \dot{m} \propto t^{-8 / 7} .
$$

Figure 1 schematically shows the inner accretion rate history across those four phases.

One can derive the time scales for phase changes. The end of Phase 0 is already given by $t_{f}$. The time when the outer disk starts to transition from non-radiative to radiative, $t_{\text {tra }}$, marks the end of Phase 1 . It is determined by $r_{o}\left(t_{\text {tra }}\right)=r_{\text {tra }}\left(t_{\text {tra }}\right) \simeq \dot{m}\left(r_{o}, t_{\text {tra }}\right)$. The third time scale $t_{\text {rad }}$ is the time when the entire disk becomes radiative, which separates Phase 2 and Phase 3. It is determined by $r_{i}=$ $r_{\text {tra }}\left(t_{\text {rad }}\right) \simeq \dot{m}\left(r_{\text {tra }}, t_{\text {rad }}\right)$. Approximate numerical estimates of these time scales are given in Figure 1. One more time scale is when the outer disk starts to transition from radiation pressure dominated to gas pressure dominated, but this does not cause a change in the inner disk accretion rate scaling, so we do not include it here. Lastly, $t_{\text {gas }}$ is the time when the entire disk becomes gas pressure dominated, setting the end of Phase 3, determined by $r_{\text {gas }}\left(t_{\mathrm{gas}}\right)=r_{i}$. A numerical estimate is $t_{\text {gas }} / t_{f}=10^{4} \sim 10^{5}$. 


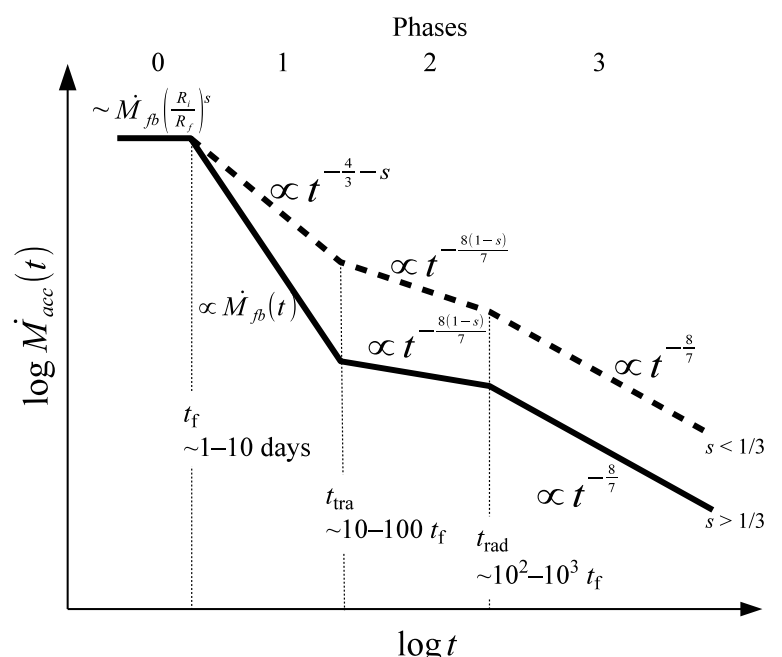

Figure 1. Schematic history of the accretion rate at the inner boundary of the TDE accretion disk across four phases. Phase 0 is the earliest, before $t_{f}$ at which the fallback rate starts to drop as $t^{-5 / 3}$. The subsequent evolution depends on the disk wind loss strength parameter $s$.

\section{DISK PRECESSION AND ITS EVOLUTION}

Most likely the captured star's angular momentum is misaligned with that of the hole. Then the accretion disk will precess due to the Lense-Thirring effect. The jet may precess as well, either because the jet orientation is intrinsically attached with the that of the disk at the jet production site, or because the outflow from the disk surface could deflect the jet. In any case, the observed luminosity would be periodically modulated by the precession. It will be interesting to investigate the disk precession and look for the signature in TDEs, especially for the well-observed jetted TDE candidate Sw J1644 + 57 which show numerous dips in the X-ray light curves and from which quasi-periodicity is claimed [17]. A few studies have investigated the possibility that the dips in the Sw J1644 X-ray light curve might be due to the disk precession $[18,19]$, but they did not consider the evolution of the disk and thus of the precession period.

Because of the strong radial dependence of the local Lense-Thirring period $t_{L T} \approx 8 \pi G M r^{3} /\left(a c^{3}\right)$, where $a$ is the $\mathrm{BH}$ dimensionless spin parameter and $r$ is the Schwarzschild-normalized orbit radius, the differential precession produces warps in the inner disk. The viscous torques due to vertical shear of warps tend to straighten out the warps, leading to the "BardeenPetterson" configuration [20]. However, if considering the propagation of warps, the above picuter changes. Especially, in the low viscosity regime $(\alpha \lesssim h / r \lesssim 1)$ which is appropriate for the TDE disk, warps propagate as waves at a speed comparable to the sound speed [21]. It is likely that $t_{\text {prop }}<t_{L T}$ is satisfid everywhere in the disk, in which case the disk can precess as a solid body [22,23].

If the disk precesses as a solid body, for a surface density profile $\Sigma \propto r^{-\zeta}$, the disk precession period can be calculated as [23]

$$
t_{\mathrm{prec}, \mathrm{disk}} \approx \frac{8 \pi}{a} \frac{G M}{c^{3}} r_{o}^{5 / 2-\zeta} r_{i}^{1 / 2+\zeta},
$$

where $r_{i}$ is the disk inner radius. For a thick, ADAF disk, $n=1 / 2$, thus, $\zeta=1 / 2-s$ (see Eq. 10). For fiducial model parameter values $a=0.9, s=0.5, r_{i}=3$ and $r_{o}=20$, a typical value for $t_{\text {prec,disk }}$ is $10^{5} M_{6} \mathrm{~s}$.

Since $t_{\text {prec,disk }}$ depends mostly on $r_{o}$ and $\zeta$ which evolve across the four phases, $t_{\text {prec,disk }}$ evolves as well. In Phase 0 , combining Eqs. (8) and (14) one finds

$$
t_{\text {prec,disk }} \propto r_{o}^{3 / 2} \propto t .
$$

In Phase 1 , similarly using the surface density profile, it is found that

$$
t_{\text {prec,disk }} \propto r_{o}^{5 / 2-\zeta} \propto \begin{cases}t^{\frac{2}{3}(2+s)}, & \text { for } s<1 / 3 \\ t, & \text { for } s>1 / 3\end{cases}
$$

In both phase 2 and phase 3 , because $r_{o}$ evolves identically in both phases with the identical $\zeta$, the $t_{\text {prec,disk }}$ evolution laws are the same:

$$
t_{\text {prec,disk }} \propto r_{o}^{3} \propto t^{6 / 7}
$$

However, we note in caution that in the last two phase the rigid precession might not be realized, at least not for the entire disk, because $h / r$ may drop to below $\alpha$.

\section{APPLICATION TO SW J1644}

The recenly discovered luminous X-ray transient Sw J1644 is identified as a jetted TDE through multiple strong evidences [24-27]. The long-term X-ray light curve of Sw J1644 is shown in the top pannel of Figure 2. It contains multiple flares before $t=5 \times 10^{5} \mathrm{~s}$, then it shows numerious dips thereafter. Disregard of these variabilities, the light curce starts a long-term power-law decline in general trend after $t \approx 10^{6} \mathrm{~s}$. The decline slope is between $t^{-5 / 3}$ and $t^{-4 / 3}$, as shown by the dashed line in the pannel. Before that time, if we neglect the significant flaring variabilities, the general trend of the light curve is approximately constant. Intrestingly, this general trend behavior resembles phases 0 and 1 in our schematic accretion rate history diagram Figure 1. Of course, this conjecture is based on the assumption that the jet luminosity scales linearly with $\dot{m}$ in a time-independent manner. If this conjecture is true, we can: (1) immediately get the fallback time scale for this event: $t_{f} /(1+z) \approx 10^{6} \mathrm{~s}$, and (2) predict that the future XRT light curve decline will flatten out at some time, as the accretion will enter phases 2 and 3 where the disk becomes radiatively efficient. The latter prediction can be tested by the XRT long-term monitoring of this event.

The middle pannel of Figure 2 shows the long-term evolution of the quasi-period in the Sw J1644 X-ray light curve obtained from [17]. The quasi-period increases with time approximately as $\propto t^{\sim 2 / 3}$. We also determine the separation times between peaks in the light curve, which are shown in the bottom pannel of Figure 2. In general trend, the peak-separation time also increases with time as $\propto t^{\sim 2 / 3}$. Those two observed timescale evolutions are both considerably slower than the predicted evolution of the disk precession period $t_{\text {prec,disk }} \propto t$. 


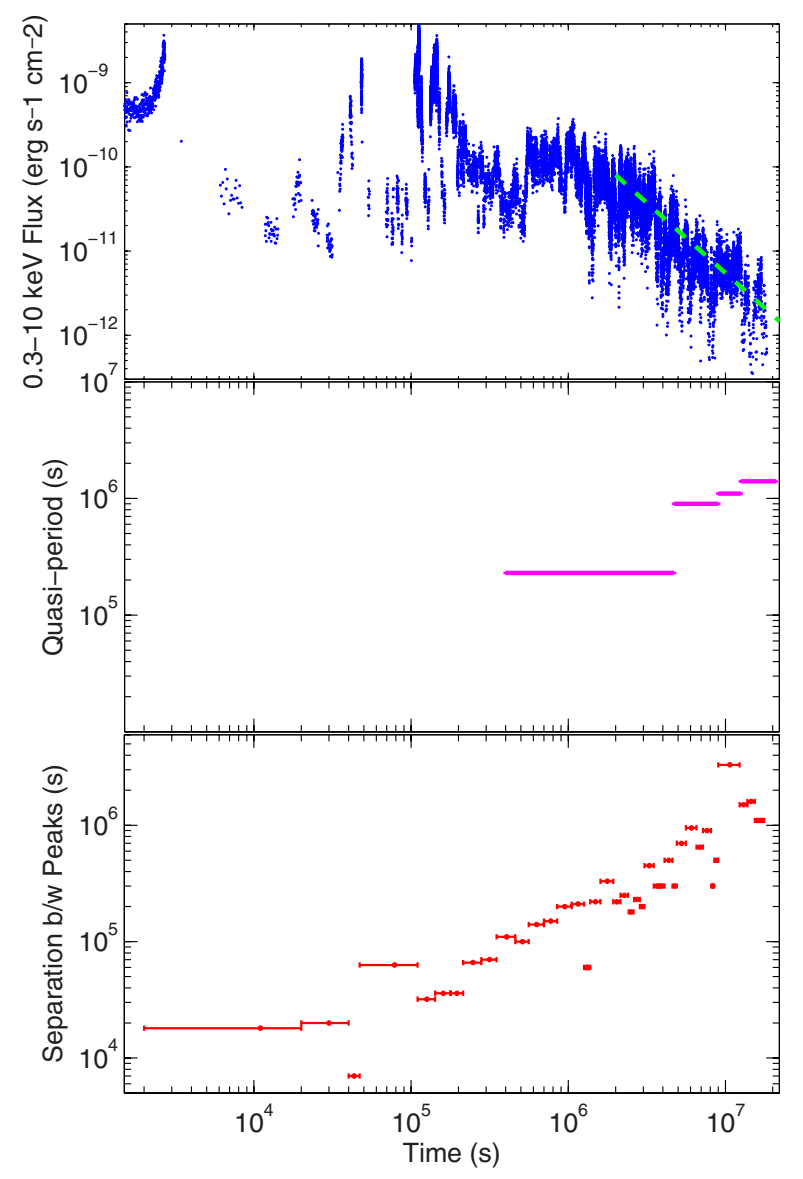

Figure 2. Top: the $0.3-10 \mathrm{keV}$ light curve of $\mathrm{Sw} \mathrm{J} 1644+57$. The dashed line is a $t^{-5 / 3}$ power law. Middle: evolution of the quasi-periods in Sw J1644 + 57 X-ray light curve found by [17]. Bottom: separations between neighboring peaks as a function of time. Identifying the peaks is done by eyes.

\section{CONCLUSIONS}

We find that the TDE accretion rate history does not necessarily track the fallback rate history, notably the $t^{-5 / 3}$ power law, when the disk evolution due to viscous spreading is carefully considered. We construct a simple analytical model that comprehensively describes the accretion rate history across 4 different phases of the disk evolution, in the presence of mass fallback and taking into account disk wind loss. Accretion rate evolves differently in those phases which are governed by how the disk heat energy is carried away, early on by advection (i.e., an ADAF) and later by radiation. The accretion rate can decline as steeply as $t^{-5 / 3}$ only if copious disk wind loss is present during the early ADAF phase. Later, the accretion rate history is $t^{-8 / 7}$ or shallower. As a by-product of our accretion rate evolution calculation, we also predict the time-dependent evolution of precession period, $t_{\text {prec,disk }}$, of the TDE accretion disk.

We apllied the analytical model to the recent jetted TDE candidate Sw J1644. Assuming a linear proportionality between the X-ray luminosity and the accretion rate, we find our model can describe the general shape of the X-ray light curve, and predict the light curve decay will become shallower at some later time. If numerious dips in the light curve of this event are to be interpreted as being modulation due to the disk precession in a solid body manner, we find the predicted disk precession period increases too fast with time compared with the observed evolution rate of dipping periods. A thorough investigation of the disk precession in the TDE context is needed to judge this interpretation of dips.

RFS thanks the organizers of the workshop for a participation travel grant.

\section{References}

[1] G. Lodato, A. R. King, J. E. Pringle, MNRAS, 392, (2009) 332

[2] G. Lodato, E. M. Rossi, MNRAS, 410, (2011) 359

[3] J. K. Cannizzo, H. M. Lee, J. Goodman, ApJ, 351, (1990) 38

[4] M. Montesinos Armijo, J. A. de Freitas Pacheco, ApJ, 736, (2011) 126

[5] M. J. Rees, Nature, 333, (1988) 523

[6] C. R. Evans, C. S. Kochanek, ApJ, 346, (1989) L13

[7] E. Ramirez-Ruiz, S. Rosswog, ApJ, 697, (2009) L77

[8] D. Lynden-Bell, J. E. Pringle, MNRAS, 168, (1974) 603

[9] J. E. Pringle, MNRAS, 248, (1991) 754

[10] B. D. Metzger, A. L. Piro, E. Quataert, MNRAS, 390, (2008) 781

[11] T. Tanaka, MNRAS, 410, (2011) 1007

[12] J. K. Cannizzo, N. Gehrels, ApJ, 700, (2009) 1047

[13] L. E. Strubbe, E. Quataert, MNRAS, 400, (2009) 2070

[14] M. A. Abramowicz, B. Czerny, J. P. Lasota, E. Szuszkiewicz, ApJ, 332, (1988) 646

[15] R. D. Blandford, M. C. Begelman, MNRAS, 303, (1999) L1

[16] R. Narayan, T. Piran, P. Kumar, ApJ, 557, (2001) 949

[17] C. J. Saxton, R. Soria, K. Wu, N. P. M. Kuin, MNRAS, 422, (2012) 1625

[18] N. Stone, A. Loeb, Phys. Rev. Lett., 108, (2012) 061302

[19] W.-H. Lei, B. Zhang, H. Gao, arXiv:1202.4231 (2012)

[20] J. M. Bardeen, J. A. Petterson, ApJ, 195, (1975) L65

[21] R. P. Nelson, J. C. B. Papaloizou, MNRAS, 309, (1999) 929

[22] R. P. Nelson, J. C. B. Papaloizou, MNRAS, 315, (2000) 570

[23] P. C. Fragile, O. M. Blaes, P. Anninos, J. D. Salmonson, ApJ, 668, (2007) 417

[24] A. J. Levan, et al., Science, 333, (2011) 199

[25] J. S. Bloom, et al., Science, 333, (2011) 203

[26] D. N. Burrows, et al., Nature, 476, (2011) 421

[27] B. A. Zauderer, et al., Nature, 476, (2011) 425 